\title{
Antiviral-Resistant Fulminant Herpes Hepatitis in Pregnancy
}

\author{
Christina A. Herrera, MD ${ }^{1}$ Kacey Y. Eichelberger, MD² Nancy C. Chescheir, MD² \\ ${ }^{1}$ Department of Obstetrics and Gynecology, University of North \\ Carolina School of Medicine, Chapel Hill, North Carolina \\ 2 Division of Maternal Fetal Medicine, University of North Carolina \\ School of Medicine, Chapel Hill, North Carolina \\ Address for correspondence Christina Herrera, MD, Department of \\ Obstetrics and Gynecology, CB\# 7570, UNC School of Medicine, Chapel \\ Hill, NC 27599-7570 (e-mail: camccall@unch.unc.edu). \\ Am J Perinatol Rep 2013;3:87-90.
}
Abstract
Keywords
- herpes hepatitis
- fulminant herpes
- pregnancy herpes
- disseminated herpes

Fulminant herpes hepatitis with disseminated extrahepatic involvement in pregnancy is rare and carries a high mortality risk. Although acyclovir remains standard first-line therapy, effective management of acyclovir-resistant disseminated herpes simplex virus (HSV) in pregnancy remains elusive. We present a case of disseminated HSV resistant to both acyclovir and foscarnet, the first double-agent resistant case in pregnancy reported in the literature to date. In this case, therapeutic delivery was the ultimate treatment resulting in full recovery.
Fulminant herpes simplex virus (HSV) hepatitis with disseminated extrahepatic involvement is a rare complication of herpes virus infection, traditionally described in significantly immunocompromised hosts. ${ }^{1}$ The immunosuppressive effects of pregnancy make pregnant women the second most common population to suffer from disseminated disease. ${ }^{2}$ Humoral and cell-mediated immunity are most depressed in the third trimester, as demonstrated by decreased T-cell count and altered B-/T-cell ratios. ${ }^{2}$ Disseminated disease is life-threatening, with maternal mortality risk reported to be as high as $39 \%{ }^{2}$ The current treatment recommendation for fulminant HSV hepatitis in pregnancy is intravenous (IV) acyclovir, with the addition of foscarnet for acyclovir-resistant cases. ${ }^{3}$ We present a case refractory to both acyclovir and foscarnet, the first of its kind reported in the literature. ${ }^{4}$ Ultimately, only delivery resulted in resolution of maternal hepatic and neurologic dysfunction.

\section{Case Report}

The patient was a 24-year-old gravida 1 with no significant medical history who presented to our hospital as a transfer of care at $27^{2 / 7}$ weeks' gestation with fever of unknown origin, hepatic failure, and leukopenia. She presented to the outside facility 7 days prior with vague complaints of fever, nausea, abdominal pain, malaise, and anorexia. At the outside hospital, she was noted to have mild transaminitis (alanine transaminase [ALT] $205 \mathrm{U} / \mathrm{L}$, aspartate transaminase [AST] $357 \mathrm{U} / \mathrm{L}$ ) and persistent fevers. Empiric broad-spectrum antimicrobial coverage was initiated, including azithromycin, piperacillin/ tazobactam, and acyclovir. Initial laboratory evaluation included negative blood cultures and hepatitis panel. Additionally, antibody testing was negative for rapid plasma reagin (RPR), human immunodeficiency virus, rubella, toxoplasma, streptococcus pneumovirus, influenza $A / B$, respiratory syncytial virus (RSV), parainfluenza, legionella, chlamydia, gonorrhea, and antinuclear antibody (ANA). Epstein-Barr virus testing was consistent with remote infection. Due to continued fevers, hypoxemia, a new chest consolidation, and worsening liver failure with associated coagulopathy, the patient was transferred to the medical intensive care unit (MICU) at our facility on hospital day 7.

Upon arrival to our MICU, physical exam was significant for mild jaundice and a few very small shallow, friable ulcerations on the labia. We obtained HSV cultures and continued broad-spectrum antibiotics with IV acyclovir from the outside facility. Despite supportive care and appropriate antiviral therapy, the patient's hepatic dysfunction worsened (AST 2290 U/L, ALT 783 U/L, lactate dehydrogenase $6551 \mathrm{U} / \mathrm{L}$, and total bilirubin $7.5 \mathrm{mg} / \mathrm{dL}$ ) with associated received

January 12, 2013 accepted after revision

February 26, 2013

published online

May 21, 2013
DOI http://dx.doi.org/

10.1055/s-0033-1343791. ISSN 2157-6998.
Copyright $\odot 2013$ by Thieme Medical Publishers, Inc., 333 Seventh Avenue, New York, NY 10001, USA. Tel: +1(212) 584-4662.
License terms

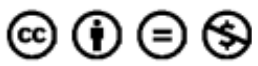


coagulopathy (international normalized ratio [INR] 1.9). On hospital day 8, she developed significant pancytopenia, hypotension requiring dual-agent pressor therapy, and acute respiratory failure requiring intubation. She had significantly altered mental status concerning for hepatic encephalopathy versus herpetic encephalitis. We diagnosed disseminated extrahepatic HSV 2 with associated fulminant hepatitis by polymerase chain reaction (PCR) in genital, liver, bronchoalveolar, blood, and cerebral spinal fluid samples. Given the lack of clinical response to acyclovir and a plateau in maternal serum HSV PCR cycles, the consulting infectious disease specialists recommended the addition of foscarnet as second-line therapy for treatment of acyclovir-resistant HSV hepatitis on hospital day 21.

Of note, initial fetal anatomic survey at the outside hospital was consistent with an isolated open-lipped schizencephaly. After extensive counseling by the maternal fetal medicine providers there, the patient opted for palliative care with no fetal monitoring or intervention. The fetal diagnosis was confirmed via ultrasound at our institution. Prior to intubation and mental status change, the patient had again confirmed her strong desire for no intervention on behalf of the fetus.

On hospital day 24 , the patient remained neurologically impaired, intubated with increasing ventilator requirements, hypotensive, and coagulopathic. Given the lack of response to dual-agent antiviral therapy, rising maternal serum lactate levels (11.2 mmol/L), and very guarded prognosis, we held a multidisciplinary conference to discuss the potential therapeutic benefit of delivery. Due to the prenatal diagnosis of schizencephaly and desire for no fetal intervention, as well as worsening maternal status, we made the decision to proceed with prostaglandin induction via misoprostol (50 $\mu \mathrm{g}$ every 6 hours). In planning for induction, we performed a fetal paracentesis due to a new finding of fetal ascites and concern for abdominal dystocia during delivery. HSV PCR on the fetal fluid returned negative. On hospital day 25 , a female fetus was delivered vaginally with no signs of life weighing 1,878 g. Fetal autopsy was declined by the patient's health care surrogate.

The patient's immediate postpartum course was complicated by transient hypofibrinogenemia concerning for disseminated intravascular coagulopathy. We placed an intrauterine balloon for 36 hours secondary to postpartum hemorrhage, which was thought to be due to coagulopathy and not related to uterine atony. The coagulopathy was resolved with factor replacement and red cell transfusion. Within 4 days of delivery, the patient was successfully extubated, weaned off pressor support, and had significant improvement in hepatic function marked by normalizing transaminases, total bilirubin, and INR. Her encephalopathy resolved more slowly, but normal mental status was noted before final discharge to home on hospital day 44. Repeat HSV PCR from blood sampling remained positive at time of discharge, but continued to trend downward. She remained on acyclovir for the duration of her hospital course and will need a prolonged outpatient course per recommendations from infectious disease specialists.

\section{Discussion}

Fulminant herpes hepatitis is rare, with the disease burden heavily concentrated among neonates and severely malnourished children. In 1969, Thomas Henry Flewett, a prominent British pathologist, and colleagues reported the first adult case in a female patient at 28 weeks' gestation who developed hepatic dysfunction following primary herpetic stomatitis. ${ }^{5}$ Since that time, 34 more cases of herpes hepatitis in pregnancy have been reported in the literature, with a mortality rate of 39\% reported by Kang and Graves in 1999, but only 9\% among the subsequent 11 cases reported from 2000 to present. ${ }^{6-14}$ This is in stark contrast to the $74.4 \%$ mortality rate for herpes hepatitis in the general population reported by Norvell et al in 2007 and may reflect the underlying disease state that made the nonpregnant patients vulnerable to disseminated herpes. ${ }^{15}$

The nomenclature used to describe variations of systemic herpes infections is applied inconsistently throughout the literature. For the purpose of our work, disseminated herpes refers to HSV documented by culture or PCR in a nongenital or mucosal fluid or tissue. Herpes hepatitis is more specific and describes patients with seropositive active herpes viremia (usually measured via serum PCR) and associated transaminitis. Fulminant herpes hepatitis includes HSV-seropositive patients with true liver failure (as evidenced by decreased albumin, elevated INR, and severe transaminitis) in critical condition. Our case describes a patient with fulminant herpes hepatitis, the most severe form of the infection.

The clinical presentation of febrile abdominal pain and anicteric hepatic dysfunction in pregnancy should prompt immediate consideration of the diagnosis of herpes hepatitis and separates this disorder from the other common etiologies of nonfebrile hepatic failure in pregnancy: acute fatty liver, preeclampsia/HELLP (hemolysis, elevated liver enzymes, and low platelets), and intrahepatic cholestasis. Clinical symptoms concerning for disseminated herpes can be nonspecific, including fever, malaise, abdominal pain, and nausea. Laboratory findings, too, can be subtle in the early stages, including mild transaminitis, leukopenia, and elevated INR. The gold standard for the diagnosis of herpes hepatitis remains liver biopsy with histopathology, electron microscopy, and culture. The classic finding of hemorrhagic necrosis, inflammation, and enlarged ground glass nuclei with marginalized chromatin is pathognomonic. ${ }^{16}$ However, percutaneous liver biopsy in the setting of maternal coagulopathy can be associated with significant bleeding-related morbidity, making alternative definitive diagnostic modalities desired. Basic serology carries a well-known risk of false negative result; PCR assay from a maternal serum sample should be performed in such cases where the diagnosis remains suspected. ${ }^{12,14}$ If the testing is positive, clinicians can follow serial PCR amplification crossing points, which correlate with viral load (the higher the cycle number, the lower the viral quantity), to determine the persistence and strength of viremia (-Fig. 1).

Though no antiviral agent has been proven effective in randomized trials, intravenous acyclovir is standard first-line therapy for presumed disseminated disease. In cases of 


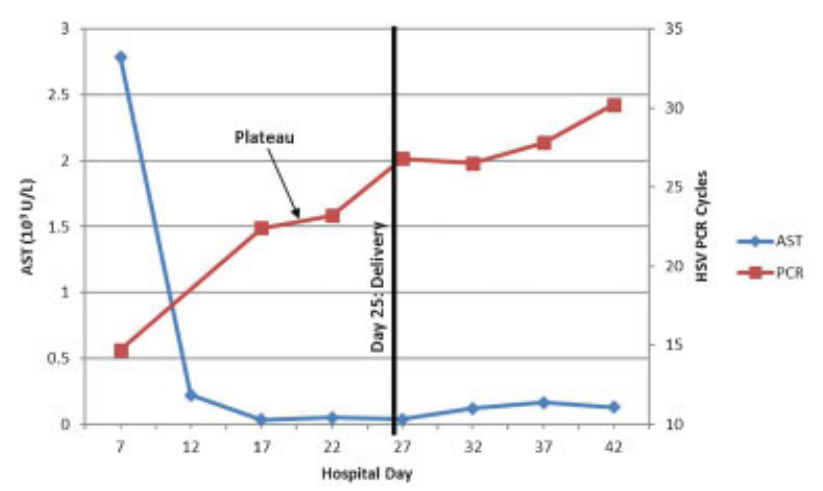

Fig. 1 Qualitative HSV PCR trending. HSV PCR cycle interpretation: The higher the cycle number, the lower the viral quantity. A change of 3.32 cycles is approximately equivalent to a change of $1 \log$ in copies per milliliter. Abbreviations: AST, aspartate aminotransferase; HSV, herpes simplex virus; PCR, polymerase chain reaction.

drug-resistant herpetic infection, intravenous foscarnet is the agent of choice but carries with it a significant risk of toxicity to the renal tubules. A pyrophosphate analogue that selectively inhibits viral polymerase, foscarnet is primarily used to treat cytomegalovirus when ganciclovir is contraindicated, as well as acyclovir-resistant herpes virus or varicella zoster. ${ }^{17,18}$ Though no adverse events have been reported with foscarnet use in pregnancy, the data are sparse, and current recommendations are for use only in the setting of a high risk of maternal mortality without treatment. ${ }^{19}$

Despite intensive supportive care and treatment with both acyclovir and foscarnet, our patient experienced progressive multiorgan dysfunction and persistent stable viremia as measured by serum qualitative HSV PCR, the first case of dual-agent resistance to be reported. When traditional intravenous antiviral therapies failed and prognosis appeared very poor, a multidisciplinary team offered the patient's family the option of therapeutic induction of labor, with the caveat that literature is sparse regarding delivery to improve innate cellmediated immune function. Fulminant herpes hepatitis is very rarely diagnosed in immunocompetent patients, and pregnant women represent a particularly vulnerable group. Though the precise mechanism for increased susceptibility has not been proven in vitro, it is well established that pregnant women have significantly depleted cell-mediated immunity as measured by helper T-cell count, which nadirs in the third trimester. ${ }^{20}$ This nadir mirrors the gestational age during which the vast majority of the aforementioned 37 cases of herpes hepatitis in pregnancy have been diagnosed. In our case, delivery occurred on hospital day 27, with significant clinical and laboratory improvement seen within 3 days postpartum.

In summary, the diagnosis of herpes hepatitis and empiric treatment with intravenous acyclovir should be strongly considered in any case of febrile hepatic dysfunction in pregnancy. We recommend consideration be given to therapeutic delivery in the setting of antiviral-resistant dis- ease with concern for maternal mortality; we also suggest that HSV qualitative PCR be used to trend disease burden.

\section{References}

1 Young EJ, Killam AP, Greene JF Jr. Disseminated herpesvirus infection. Association with primary genital herpes in pregnancy. JAMA 1976;235:2731-2733

2 Kang AH, Graves CR. Herpes simplex hepatitis in pregnancy: a case report and review of the literature. Obstet Gynecol Surv 1999;54:463-468

3 Workowski KA, Berman S; Centers for Disease Control and Prevention (CDC). Sexually transmitted diseases treatment guidelines, 2010. MMWR Recomm Rep 2010;59(RR-12):1-110

4 Gupta R, Warren T, Wald A. Genital herpes. Lancet 2007;370: 2127-2137

5 Flewett TH, Parker RG, Philip WM. Acute hepatitis due to herpes simplex virus in an adult. J Clin Pathol 1969;22:60-66

6 Mortelé KJ, Barish MA, Yucel KE. Fulminant herpes hepatitis in an immunocompetent pregnant woman: $\mathrm{CT}$ imaging features. Abdom Imaging 2004;29:682-684

7 Nagappan R, Parkin G, Simpson I, Sievert W. Fulminant hepatic failure from herpes simplex in pregnancy. Med J Aust 2002; 176:595-596

8 Allen RH, Tuomala RE. Herpes simplex virus hepatitis causing acute liver dysfunction and thrombocytopenia in pregnancy. Obstet Gynecol 2005;106(5 Pt 2):1187-1189

9 Lazar P. An interesting herpes simplex infection. Arch Dermatol 1977;113:145-149

10 Frederick DM, Bland D, Gollin Y. Fatal disseminated herpes simplex virus infection in a previously healthy pregnant woman. A case report. J Reprod Med 2002;47:591-596

11 Halliday J, Lokan J, Angus PW, Gow P. A case of fulminant hepatic failure in pregnancy. Hepatology 2010;51:341-342

12 Thurman RH, König K, Watkins A, Weerasiri T, Permezel M. Fulminant herpes simplex virus hepatic failure in pregnancy requiring liver transplantation. Aust $\mathrm{N} \mathrm{Z} \mathrm{J} \mathrm{Obstet} \mathrm{Gynaecol}$ 2010;50:492-494

13 Navaneethan U, Lancaster E, Venkatesh PG, Wang J, Neff GW. Herpes simplex virus hepatitis-it's high time we consider empiric treatment. J Gastrointestin Liver Dis 2011;20:93-96

14 Nienaber JH, McNamara DR, Banerjee R, Pritt BS, Karre T, Sohail MR. Fulminant gestational hepatitis due to primary herpes simplex type 2 infection: use of serum HSV polymerase chain reaction for noninvasive diagnosis. Diagn Microbiol Infect Dis 2012;72:181-184

15 Norvell JP, Blei AT, Jovanovi BD, Levitsky J. Herpes Simplex Virus hepatitus: an analysis of the published literature and institutional cases. Liver Transpl 2007;10:1428-34

16 Kaufman B, Gandhi SA, Louie E, Rizzi R, Illei P. Herpes simplex virus hepatitis: case report and review. Clin Infect Dis 1997;24:334-338

17 Viera MH, Amini S, Huo R, Konda S, Block S, Berman B. Herpes simplex virus and human papillomavirus genital infections: new and investigational therapeutic options. Int $\mathrm{J}$ Dermatol 2010;49:733-749

18 Razonable RR. Antiviral drugs for viruses other than human immunodeficiency virus. Mayo Clin Proc 2011;86:1009-1026

19 Money D, Steben M; Infectious Diseases Committee, Society of Obstetricians and Gynecologists of Canada. Guidelines for the management of herpes simplex virus in pregnancy. J Obstet Gynaecol Can 2008;30:514-526

20 Sridama V, Pacini F, Yang SL, Moawad A, Reilly M, DeGroot LJ. Decreased levels of helper T cells: a possible cause of immunodeficiency in pregnancy. N Engl J Med 1982;307:352-356 